\title{
DESENVOLVIMENTO E ACEITABILIDADE DE MUFFINS ADICIONADOS DE FARINHA DE CASCA DE UVA DAS CULTIVARES ANCELOTTA E BORDÔ
}

Gabriela Datsch BENNEMANN ${ }^{1}$

Marina de Campos NEZELLO ${ }^{2}$

Karolyne Kruger Carvalho EING ${ }^{3}$

Daiana NOVELLO ${ }^{4}$

Kélin SCHWARZ

Renato Vasconcellos BOTELHO ${ }^{6}$

\begin{abstract}
${ }^{1}$ Nutricionista formada pela Universidade Federal do Paraná. Doutoranda no programa de pós-graduação em Agronomia da Universidade Estadual do Centro - Oeste / Guarapuava-PR. Docente do Departamento de Nutrição da Universidade Estadual do Centro - Oeste / Guarapuava-PR.
\end{abstract}

${ }^{2}$ Graduanda em Nutrição, Universidade Estadual do Centro-Oeste/ Guarapuava-PR.

${ }^{3}$ Nutricionista formada pela Universidade Estadual do Centro-Oeste/Guarapuava-PR. Mestranda no programa de pósgraduação em Agronomia da Universidade Estadual do Centro - Oeste / Guarapuava-PR.

${ }^{4}$ Nutricionista formada pela Universidade Estadual do Centro-Oeste/Guarapuava-PR.Doutora em Ciências(Energia Nuclearna Agricultura e no Ambiente) pelo Centro de Energia Nuclear na Agricultura (CENA) DA Universidade de São Paulo (USP). Docente do Departamento de Nutrição da Universidade Estadual do Centro - Oeste / Guarapuava-PR.

${ }^{5}$ Nutricionista formada pela Universidade Regional do Noroeste do Estado do Rio Grande do Sul - UNIJUÍ. Doutora em Tecnologia de Alimentos pela Universidade Estadual de Campinas - UNICAMP. Docente do Departamento de Nutrição da Universidade Estadual do Centro - Oeste / Guarapuava-PR.

${ }^{6}$ Engenheiro Agrônomo, formado pela Faculdade de Ciências Agronômicas - UNESP/Botucatu-SP. PósDoutor Agronomia na seção de Fruticultura do Instituto Agronômico de Campinas e na Escola de Agrárias e Veterinária da Universidade de Bolonha, Itália. Docente do Departamento de Agronomia da Universidade Estadual do Centro - Oeste / Guarapuava-PR.

Recebido em: 12/04/2016 - Aprovado em: 15/10/2016 - Disponibilizado em: 18/12/2016

\section{RESUMO:}

O estudo objetivou verificar a aceitabilidade sensorial de muffins adicionados de farinha de casca de uva (FCU) das cultivares Ancellotta e Bordô, bem como determinar a atividade microbiológica destas farinhas. Foram elaboradas cinco formulações de muffins, sendo: F1: padrão (0\%) e as demais adicionadas de 25\% (F2) e 50\% (F3) de FCU da cultivar Ancellotta; $25 \%$ (F4) e 50\% (F5) da FCU da cultivar Bordô. Participaram da análise sensorial 50 provadores, não treinados, com idade acima de 18 anos. A partir da análise sensorial, percebeu-se que as maiores notas para F1 e F2 foram verificadas nos atributos aparência, cor e aceitação global. Porém, destaca-se que as amostras F2 e F4 foram aquelas com maior aceitabilidade, comparadas à F3 e F5. Em relação à atividade microbiológica, não foram encontrados valores significativos, assegurando os critérios de controle de qualidade das amostras. Conclui-se que a adição de até $25 \%$ de FCU de ambos as cultivares foi bem aceito pelos provadores.

Palavras chave: Reaproveitamento. Resíduos. Vinicultura. 


\title{
DEVELOPMENT AND ACCEPTABILITY OF MUFFINS ADDED WITH GRAPE SKIN FLOUR OF CULTIVARS ANCELLOTTA AND BORDÔ
}

\begin{abstract}
:
The study aimed to verify the sensory acceptability of muffins added of grape skin flour (FCU) of Ancellotta and Maroon cultivars, and to determine the microbiological activity of these flours. Five formulations of muffins were prepared, as follows: F1: Standard (0\%) and the other added 25\% (F2) and 50\% (F3) of FCU cultivar Ancellotta; 25\% (F4) and $50 \%$ (F5) of the cultivation FCU bordô. 50 tasters participated of sensorial analysis, untrained, aged above 18 years. From the sensory evaluation, it was observed that the highest scores for F1 and F2 were found in the attributes appearance, color and overall acceptability. However, it is emphasized that the F2 and F4 samples were those with greater acceptability compared to the F3 and F5. Regarding the microbiological activity, significant values were not found, ensuring the quality of the samples control criteria. It is concluded that addition of up to $25 \%$ of FCU two cultivars was well accepted by the panelists
\end{abstract}

Keywords: Reuse. Waste. Viniculture.

\section{INTRODUÇÃO}

A produção de uva (viticultura) representa boa parte da plantação de frutas no mundo, sendo 60 milhões de toneladas cultivados anualmente, tendo como França, Itália, EUA e China os principais produtores. Entretanto, cerca de $80 \%$ da cultura da uva é dedicada à produção de vinhos e derivados (FAO, 2005), o que resulta em aproximadamente 19 milhões de toneladas de resíduos (TEIXEIRA, 2010).

Um subproduto da indústria vinícola é o bagaço (uma mistura das cascas e sementes), $\quad$ o qual representa aproximadamente $20 \%$ das uvas colhidas (ROCKENBACH, 2008). Este material apresenta elevados teores de compostos bioativos como os ácidos fenólicos $(219,56$ a $\left.1242,78 \mathrm{mg} 100 \mathrm{~g}^{-1}\right)$, antiocianinas $(7,02 \mathrm{a}$ $\left.82,15 \mathrm{mg} 100 \mathrm{~g}^{-1}\right)$ e flavonóides $(19,18$ e 37,75 $\mathrm{mg} 100 \mathrm{~g}^{-1}$ ) caracterizando-o como uma boa fonte de antioxidantes naturais, além de apresentar elevado conteúdo de fibras
(12,56\%). Além disso, o bagaço da uva apresenta um alto teor de calorias, carboidratos $(19,46 \%)$ e lipídeos $(1,42 \%)$, sendo estes compostos principalmente por gorduras insaturadas (ácido linoleico $(0,085 \%)$ e oleico (1,335\%). Destaca-se também, seu elevado teor em vitamina $\mathrm{E}$ (1,764 \%) (LOULI et al., 2004; PINELO et al., 2005; ABE et al., 2007; LAFKA et al., 2007; MAKRIS et al., 2007; SOARES et al., 2008; SPANGHERO et al., 2009; NÚCLEO

\section{DE ESTUDO E PESQUISAS EM} ALIMENTAÇÃO, 2011).

Natividade et al. (2010), analisando a composição centesimal de farinhas elaboradas com resíduo do processamento de suco de uva, de 3 cultivares diferentes, entre elas a Bordô, revelou que esta farinha possui 10,29\% de umidade, $5,62 \%$ de proteína, $3,09 \%$ de cinzas, $5,04 \%$ de fibra solúvel, $49,59 \%$ de fibra insolúvel e possui um alto teor calórico $(153,08 \mathrm{kcal}$ em $100 \mathrm{~g}$ de 
farinha). Já a cultivar Ancellotta faz parte da espécie Vitis vinífera. É uma uva originária da Itália, a qual foi introduzida na produção de uvas da Serra Gaúcha recentemente, no ano de 2000. Apresenta elevado conteúdo de matéria corante, e por este motivo teve rápida expansão, sua maior utilização é em cortes de vinhos de outras cultivares para melhorar sua coloração (CAMARGO, 2005 - 2007). A cultivar Bordô, pertence ao grupo de uvas da espécie americana Vitis labrusca, o qual representa mais de $80 \%$ da produção brasileira, utilizada principalmente para produção de vinho e sucos (CAMARGO, 2015).

A procura do consumidor por alimentos que tragam benefícios à saúde estimula novos processos de produção, que gerem baixo volume de resíduos sólidos ou que proporcionem seu reaproveitamento (ROCKENBACH, 2008). Neste contexto o muffin é caracterizado por ser um produto de panificação, feito para ser individual e de rápido consumo, são semelhantes ao cupcakes em tamanho e tempo de preparo. Podem possuir vários sabores, tanto salgados, quanto doces. Bolos industrializados, de tamanho pequeno, em embalagens individuais, são uma forte tendência no mercado, para consumo rápido, em intervalos e lanches, principalmente os destinados ao público infantil (PAVANELLI et al., 2000).

A partir desta realidade, é sabido que a procura e o consumo de produtos enriquecidos, é recorrente, sendo assim, relevante para a indústria de alimentos, pois beneficiam o consumidor com o alto teor nutritivo destes alimentos (FROTA et al., 2009). E através da análise sensorial que abrange técnicas e métodos com a finalidade de detectar a reação dos sentidos e as propriedades sensoriais, como: cor, aroma e sabor dos alimentos. A partir disto, torna-se viável o desenvolvimento de novos produtos. (FERREIRA et al., 2000; NASCIMENTO et al., 2009).

A análise microbiológica é um dos parâmetros mais importantes que indicam a qualidade e a inocuidade de alimentos. Pois os alimentos estão sujeitos a conter microrganismos contaminantes, estes são capazes de causar alterações indesejáveis nos alimentos, e nas pessoas que os consomem, podendo assim serem patogênicos. Por este motivo é necessária a análise microbiológica para a aquisição de informações sobre a produção do alimento e as condições de higiene durante a produção, processamento, armazenamento e distribuição para o consumo do mesmo (ANVISA, 2001).

O objetivo deste estudo foi avaliar a aceitabilidade sensorial de muffins adicionados de Farinha de Casca de Uva (FCU) das cultivares Ancellotta e Bordô, bem como determinar a atividade microbiológica das farinhas. 


\section{MATERIAL E MÉTODOS}

\section{Aquisição da matéria-prima}

Foram utilizados $20 \mathrm{~kg}$ de bagaço de ambas as uvas, sendo a cultivar Bordô, proveniente da Vinícola Dell Mont, localizada no Município de Bituruna, no sul do Estado do Paraná, situada a uma altitude de 865 m, e a cultivar Ancellotta, proveniente da Vinícola Villagio Grando, localizada no Município de Água Doce, Norte do Estado de Santa Catarina, situada a $969 \mathrm{~m}$ de altitude. As amostras foram coletadas após a abertura dos tanques de fermentação, sendo imediatamente acondicionados em caixas térmicas com gelo para serem transportados em temperatura de 2 a $6^{\circ} \mathrm{C}$ até a cidade de Guarapuava, PR. Os demais ingredientes utilizados na elaboração foram adquiridos no comércio local da cidade de Guarapuava.

\section{Preparo da farinha de casca de uva}

As amostras foram processadas no Laboratório de Fruticultura do Curso de PósGraduação em Agronomia do Campus Cedeteg da UNICENTRO. Inicialmente, o bagaço de cada uma das cultivares, foi acomodado em bandejas de alumínio (750 $\mathrm{mL}$ ), com espessura de aproximadamente 3 $\mathrm{cm}$. O bagaço foi então submetido à secagem em estufa com circulação e renovação de ar (Solab, Brasil) à $45^{\circ} \mathrm{C}$, até atingir a umidade de $<14 \% \mathrm{p} / \mathrm{p}$ indicada para a produção de FCU (AOAC, 2011). Após, permaneceram em temperatura ambiente $\left(22^{\circ} \mathrm{C}\right)$ até total resfriamento. Foi realizada separação e padronização granulométrica das sementes e cascas por meio de agitação em conjunto de peneiras do tipo Tyler com aberturas de malhas de $3,327 \mathrm{~mm}, 2,362 \mathrm{~mm}$ e $1,651 \mathrm{~mm}$ (Tamis, Brasil). Em seguida, as cascas foram trituradas em moinho rotor (Marconi, Brasil), até a obtenção da FCU, com rendimento de $1,2 \mathrm{~kg}$ e 1,6 kg de FCU das cultivares Bordô e Ancellotta respectivamente.

\section{Formulações}

As formulações foram preparadas individualmente no Laboratório de Técnica Dietética do Departamento de Nutrição da UNICENTRO. A pesagem de todos os ingredientes foi realizada em balança digital (Filizola $^{\circledR}$, Brasil) com precisão de $0,1 \mathrm{~g}$ e capacidade máxima de $15 \mathrm{~kg}$.

Foram elaborados 5 tipos de muffins, sendo: F1: padrão $(0 \%)$ e as demais adicionadas de $25 \%(\mathrm{~F} 2)$ e $50 \%$ (F3) de FCU da cultivar Ancellotta; 25\% (F4) e 50\% (F5) da FCU da cultivar Bordô. Estes níveis de adição foram definidos por meio de testes sensoriais preliminares realizados com o produto. Além das porcentagens de FCU, foram utilizados os ingredientes descritos na Tabela 1. 
Tabela 1 - Ingredientes das formulações de muffins adicionados de farinha da casca de uva (FCU)

\begin{tabular}{|c|c|c|c|c|c|c|c|c|c|c|}
\hline \multirow[t]{2}{*}{ Ingredientes } & \multicolumn{2}{|c|}{ F1 } & \multicolumn{2}{|c|}{ F2 } & \multicolumn{2}{|c|}{ F3 } & \multicolumn{2}{|c|}{ F4 } & \multicolumn{2}{|c|}{ F5 } \\
\hline & g & $\%$ & $\mathbf{G}$ & $\%$ & $\mathbf{g}$ & $\%$ & $\mathbf{g}$ & $\%$ & $\mathbf{g}$ & $\%$ \\
\hline Farinha de trigo & $\frac{8}{155}$ & 32,94 & 116,25 & 24,7 & 77,5 & 16,47 & 116,25 & 24,7 & 77,5 & 16,47 \\
\hline Leite integral & 120 & 25,5 & 120 & 25,5 & 120 & 25,5 & 120 & 25,5 & 120 & 25,5 \\
\hline Açúcar refinado & 90 & 19,13 & 90 & 19,13 & 90 & 19,13 & 90 & 19,13 & 90 & 19,13 \\
\hline Manteiga sem sal & 50 & 10,63 & 50 & 10,63 & 50 & 10,63 & 50 & 10,63 & 50 & 10,63 \\
\hline Ovo inteiro & 50 & 10,63 & 50 & 10,63 & 50 & 10,63 & 50 & 10,63 & 50 & 10,63 \\
\hline Fermento biológico & 4 & 0,85 & 4 & 0,85 & 4 & 0,85 & 4 & 0,85 & 4 & 0,85 \\
\hline Sal & 1,5 & 0,32 & 1,5 & 0,32 & 1,5 & 0,32 & 1,5 & 0,32 & 1,5 & 0,32 \\
\hline FCU & 0 & 0 & 38,75 & 8,23 & 77,5 & 16,47 & 38,75 & 8,23 & 77,5 & 16,47 \\
\hline
\end{tabular}

Para a elaboração dos muffins, a manteiga e o açúcar foram misturados em uma batedeira doméstica (Britânia, Brasil), por 5 minutos. Em seguida, foram acrescidos os ovos e misturados até homogeneização. Logo após foram adicionadas as farinhas, o leite e o sal, sendo misturados manualmente por 10 minutos. Por último, adicionou-se o fermento, o qual foi misturado até homogeneização.

A massa foi disposta em formas de alumínio (3 $\mathrm{cm}$ de diâmetro) e assadas em forno convencional pré-aquecido (Consul, Brasil), em temperatura média $\left(180^{\circ} \mathrm{C}\right)$, por aproximadamente 15 minutos.

\section{Análise sensorial}

Participaram da pesquisa 50 provadores não treinados, de ambos os gêneros, com idade entre 18 e 73 anos, recrutados na Feira do Agricultor do Campus CEDETEG da UNICENTRO, Guarapuava, PR. Os produtos foram submetidos à análise sensorial em uma sala próxima a feira. Cada prova foi realizada individualmente, sendo que o provador foi orientado para $o$ preenchimento das respostas. Foram avaliados os atributos de aparência, aroma, sabor, textura e cor, por meio de uma escala hedônica estruturada mista de 9 pontos variando de 1 ("Gostei muitíssimo") a 9 ("Desgostei muitíssimo"). Foram aplicadas também questões de aceitação global e intenção de compra analisadas através de uma escala estruturada de 5 pontos (1 "gostei muito"/ "compraria com certeza a 5 "desgostei muito/ "não compraria") (DUTCOSKI, 2011).

Os julgadores receberam uma porção de cada amostra (aproximadamente 10 g), em formas de papel descartáveis brancas, codificados com números de três dígitos, de forma casualizada e balanceada, acompanhados de água para realização do branco. As formulações foram oferecidas aos julgadores de forma simultânea.

\section{Índice de aceitabilidade (IA)}

O cálculo do IA das formulações foi realizado segundo a fórmula: $I A(\%)=A x$ 100/B (A = nota média obtida para o produto; $B=$ nota máxima dada ao produto) (DUTCOSKI, 2011). 


\section{Avaliação microbiológica}

As FCU das cultivares Ancellotta e Bordô foram analisadas em relação à contagem de coliformes termotolerantes e contagem de bolores e leveduras a $45^{\circ} \mathrm{C}$ de acordo com as metodologias propostas pela American Public Health Association, Association of Analytical Communities e Association of Official Analytical Chemists (APHA, 2001; AOAC, 2012).

Para a realização da análise das amostras foi levado em conta suas características, de acordo com a Resolução RDC n ${ }^{\circ} 12$ de 2001 da ANVISA.

\section{Análise estatística}

Os dados foram analisados com auxílio do software Statgraphics Plus ${ }^{\circledR}$, versão 5.1, através da análise de variância (ANOVA). A comparação de médias foi realizada pelo teste de Tukey, com nível de $5 \%$ de significância.

\section{Questões éticas}

Este trabalho foi aprovado pelo Comitê de Ética em Pesquisa da UNICENTRO, parecer número $\mathrm{n}^{\circ}$ 1.093.293 de 2015. Como critérios de exclusão foram considerados os seguintes fatores: possuir idade inferior a 18 anos, recusar a assinar o Termo de Consentimento Livre e Esclarecido (TCLE) e fichas de análise sensorial preenchidas de forma errada ou incompleta.

\section{RESULTADOS E DISCUSSÃO}

\section{Avaliação microbiológica}

As contagens de coliformes termotolerantes e de bolores e leveduras a 45 - C das FCU das cultivares Ancellotta e Bordô foram de $<10^{2} \mathrm{UFC} / \mathrm{g}$ para coliformes termotolerantes e $<10^{3} \mathrm{UFC} / \mathrm{g}$ para bolores e leveduras a $45^{\circ} \mathrm{C}$. Ambas as avaliações apresentaram-se de acordo com os parâmetros de adequação exigidos pela Agência Nacional de Vigilância Sanitária - ANVISA (BRASIL, 2001). Assim como no estudo de Ferreira et al (2010) que obteve resultados semelhantes, abaixo dos limites recomendados pela ANVISA, em FBU, para utilização em cereais matinais.

\section{Análise sensorial}

Por meio da Tabela 2 pode-se verificar o resultado da avaliação sensorial dos muffins padrão e adicionadas de FCU. 
Tabela 2 - Médias do índice de aceitabilidade (IA) e dos testes sensoriais afetivos e intenção de compra, realizados para muffins padrão e adicionados de farinha da casca de uva das cultivares Ancellotta e Bordô

\begin{tabular}{|c|c|c|c|c|c|}
\hline & & \multicolumn{2}{|c|}{ Ancellotta } & \multicolumn{2}{|c|}{ Bordô } \\
\hline & $0 \%$ & $25 \%$ & $50 \%$ & $25 \%$ & $50 \%$ \\
\hline Formulações/ & F1 & F2 & F3 & F4 & F5 \\
\hline Atributos & Média $\pm E P M$ & Média $\pm E P M$ & Média $\pm E P M$ & Média $\pm E P M$ & Média $\pm E P M$ \\
\hline Aparência & $7,80 \pm 0,23^{\mathrm{a}}$ & $7,38 \pm 0,24^{\mathrm{a}}$ & $5,90 \pm 0,31^{b}$ & $6,52 \pm 0,28^{c}$ & $4,68 \pm 0,30^{d}$ \\
\hline $\mathrm{IA}(\%)$ & 86,67 & 82,00 & 65,56 & 72,44 & 52,00 \\
\hline Aroma & $7,78 \pm 0,23^{\mathrm{a}}$ & $6,70 \pm 0,24^{\mathrm{b}}$ & $5,60 \pm 0,26^{\mathrm{c}}$ & $6,80 \pm 0,21^{\mathrm{b}}$ & $5,82 \pm 0,27^{\mathrm{c}}$ \\
\hline IA $(\%)$ & 86,44 & 74,44 & 62,20 & 75,56 & 64,67 \\
\hline Sabor & $7,94 \pm 0,20^{\mathrm{a}}$ & $6,90 \pm 0,24^{\mathrm{b}}$ & $5,34 \pm 0,30^{\mathrm{c}}$ & $6,84 \pm 0,22^{b}$ & $5,38 \pm 0,31^{\mathrm{c}}$ \\
\hline IA $(\%)$ & 82,22 & 76,67 & 59,33 & 76,00 & 59,78 \\
\hline Textura & $7,96 \pm 0,19^{\mathrm{a}}$ & $6,90 \pm 0,24^{b}$ & $5,36 \pm 0,29^{c}$ & $6,82 \pm 0,22^{b}$ & $5,38 \pm 0,31^{\mathrm{c}}$ \\
\hline IA (\%) & 86,67 & 76,67 & 58,22 & 75,78 & 59,78 \\
\hline Cor & $7,80 \pm 0,23^{\mathrm{a}}$ & $7,42 \pm 0,24^{\mathrm{a}}$ & $5,90 \pm 0,31^{\mathrm{b}}$ & $6,50 \pm 0,28^{\mathrm{c}}$ & $4,66 \pm 0,29^{\mathrm{d}}$ \\
\hline IA $(\%)$ & 86,67 & 82,44 & 56,33 & 72,22 & 51,78 \\
\hline Aceitação global & $7,78 \pm 0,21^{\mathrm{a}}$ & $7,28 \pm 0,22^{\mathrm{a}}$ & $5,56 \pm 0,29^{\mathrm{b}}$ & $6,70 \pm 0,22^{c}$ & $5,48 \pm 0,26^{\mathrm{b}}$ \\
\hline $\mathrm{IA}(\%)$ & 86,44 & 80,89 & 61,78 & 74,44 & 60,89 \\
\hline Intenção de compra & $4,32 \pm 0,13^{\mathrm{a}}$ & $3,72 \pm 0,16^{\mathrm{b}}$ & $2,56 \pm 0,17^{\mathrm{c}}$ & $3,48 \pm 0,15^{\mathrm{b}}$ & $2,58 \pm 0,20^{\mathrm{c}}$ \\
\hline IA $(\%)$ & 86,40 & 74,40 & 51,20 & 69,60 & 51,60 \\
\hline
\end{tabular}

Maiores notas $(\mathrm{p}<0,05)$ para F1 e F2 foram verificadas nos atributos aparência, cor e aceitação global. Já para os atributos aroma, sabor, textura e intenção de compra houve maior aceitação da F1. Segundo Rocha (2011), este efeito é explicado, pois o bagaço de uva possui elevados teores de substâncias fenólicas, as quais são compostas por um anel aromático e que, quando acrescidos em grandes quantidades, podem proporcionar sabor amargo aos produtos, reduzindo sua aceitação. No entanto, Piovesana et al. (2013) avaliando amostras de cookies acrescidos de Farinha de Bagaço de UVA (FBU) (15, 20 e $25 \%$ ) verificaram boa aceitação para os produtos, não houve formulação padrão no estudo, mas a formulação com a menor concentração foi a mais aceita.

As amostras F2 e F4 (FCU Ancellotta) obtiveram maior aceitabilidade, no geral, comparadas à F3 e F5 (FCU Bordô), em todos atributos.

Apenas as formulações F1, F2 e F4 apresentaram IA maior que $70 \%$ na maioria dos atributos, exceto para F4 (intenção de compra), o que classifica estes produtos com boa aceitabilidade sensorial (TEIXEIRA et al., 1987). Os resultados encontrados por Bender (2015) apresentaram-se contrários aos do presente estudo, que preparou muffins com adição de FBU. O mesmo obteve um bom IA para todas as formulações dos muffins produzidos com FCU, com a adição de teores crescentes de farinha de casca de uva Tannat $(5,7,5$ e $10 \%)$, isto pode ocorrer devido as menores concentrações realizadas e também a cultivar que é diferente do presente estudo.

A figura 1 apresenta a distribuição dos provadores pelos valores hedônicos para cada atributo sensorial. 

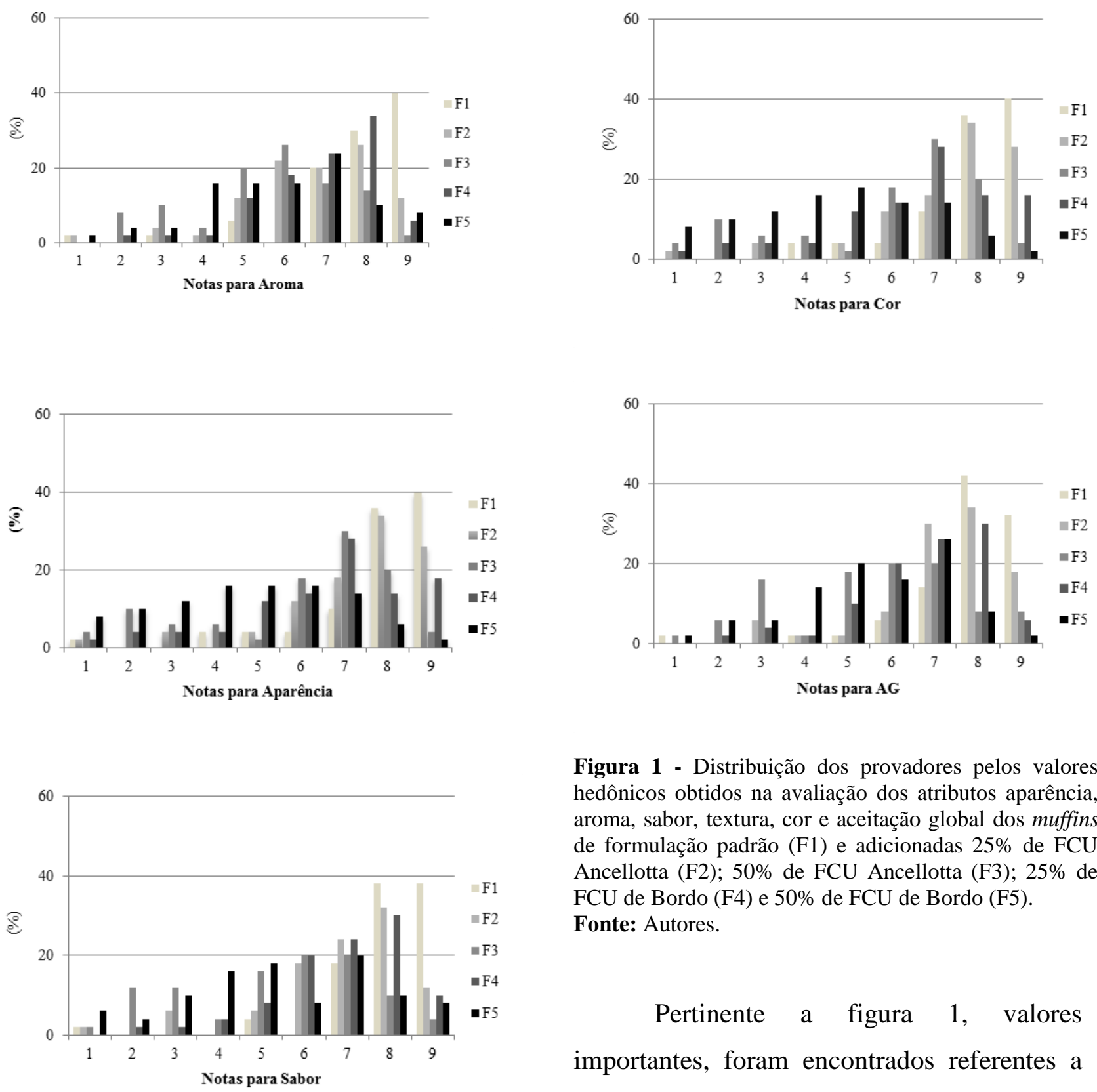

Figura 1 - Distribuição dos provadores pelos valores hedônicos obtidos na avaliação dos atributos aparência, aroma, sabor, textura, cor e aceitação global dos muffins de formulação padrão (F1) e adicionadas $25 \%$ de FCU Ancellotta (F2); $50 \%$ de FCU Ancellotta (F3); 25\% de FCU de Bordo (F4) e 50\% de FCU de Bordo (F5).

Fonte: Autores.

Pertinente a figura 1, valores importantes, foram encontrados referentes a característica aparência para F1 (padrão) e F2 (25\% Ancellotta), que obtiveram nota 8 , para

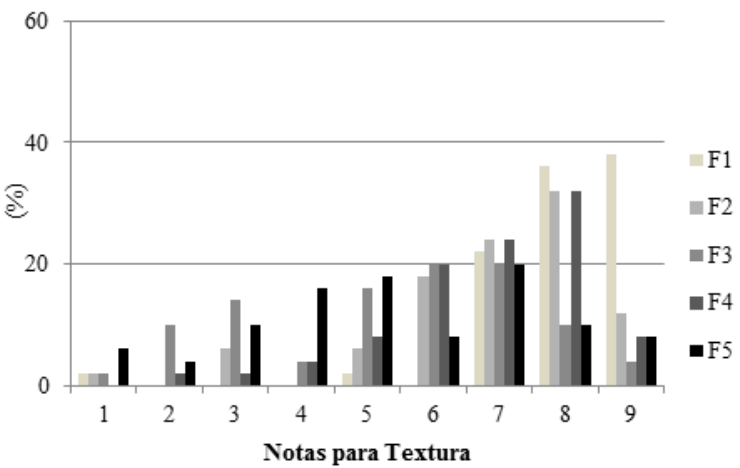
aproximadamente $40 \%$ dos provadores. Ao avaliar o atributo aroma, outro dado interessante, foi verificado na $\mathrm{F} 4 \quad(50 \%$ Ancellotta), que obteve nota 8 para, em torno de $35 \%$ dos testadores, valor maior que o obtido por F1. No que diz respeito ao atributo cor, F1 e F2 mantiveram nota 8 para aproximadamente $35 \%$ dos testadores. Referente à aceitação global, F1 alcançou 
nota 8 e 9 para em torno de $40 \%$ e $30 \%$ do provadores, respectivamente.

Entretanto, para as amostras F3 e F5, as notas se concentraram abaixo de 5 (Figura 1). No estudo de Bender (2015), a análise sensorial foi realizada por meio de escala hedônica de 7 pontos, no entanto é possível observar que os avaliadores pontuaram as melhores notas para a menor concentração (5\%) para as menores concentrações das farinhas da uva Riesling. Paras o muffins fabricados a partir da farinha da casca de uva Tannat, verificou-se as maiores pontuações para todos os quesitos, para maior concentração utilizada (10\%). Já no estudo de Oliveira et al. (2009), em que foi desenvolvido cereal matinal enriquecido com farinha de casca com semente de uva, das cultivares Bordô e Isabel. Observou-se, ao contrário do presente estudo, quanto maior a concentração da farinha, maior foi a aceitabilidade dos provadores.

É conhecido que na casca da uva encontram-se elevados teores de taninos condensados, o que contribui fortemente para a atividade antioxidante, porém estes compostos são responsáveis pela adstringência nos alimentos (GRUZ, et al 2013). Desta forma, podem ter influenciado de forma indesejável as propriedades sensoriais das farinhas e consequentemente dos muffins.

\section{CONCLUSÃO}

A elaboração dos produtos permitiu comprovar que um nível de adição de até $25 \%$ de farinha da casca de uva em muffins foi bem aceito pelos provadores. Assim sendo, a farinha de casca de uva pode ser considerada um potencial ingrediente para adição em muffins e similares, podendo ser oferecidos com altas expectativas de aceitação. Principalmente a FCU da cultivar Ancellotta que obteve maior aceitabilidade em relação a FCU da cultivar Bordo.

Os produtos apresentaram-se microbiologicamente seguros para o consumo e de acordo com a recomendação da legislação brasileira.

\section{REFERÊNCIAS}

AOAC International. Official Methods of Analysis of AOAC International. 18 ed. 4 rev. Gaithersburg: AOAC, 2011. 1505p.

ABE, L.T.; MOTA, R.V.; LAJOLO, F.M.; GENOVESE, M.I. Compostos fenólicos e capacidade antioxidante de cultivares de uvas Vitis labrusca L. e Vitis vinifera L. Ciência e Tecnologia de Alimentos, Campinas, v. 27, n. 2, p.394-400, abr. 2007.

AGÊNCIA NACIONAL DE VIGILÂNCIA SANITÁRIA. RDC N ${ }^{\circ}$ 171: Resolução RDC $\mathrm{n}^{\circ} 12$, de 02 de janeiro de 2001. Brasil: D.O.U. - Diário Oficial da União; Poder Executivo, 2001. 48 p.

ASSOCIAÇÃO BRASILEIRA DE NORMAS TÉCNICAS - ABNT. NBR 12994: Métodos de Análise Sensorial dos Alimentos e Bebidas. Rio de Janeiro: ABNT, 1993. p.2. 
BENDER, A.B.B.; SPERONI, C.S.; SILVA, L.P. Fibra alimentar a partir de casca de uva: Desenvolvimento e incorporação em bolos tipo muffins. 2015. 132 f. Dissertação

(Mestrado do Programa de Pós-Graduação em Ciências e Tecnologia dos Alimentos) -

Universidade Federal de

CAMARGO, U.A. (Brasília). Agência Embrapa de Informação Tecnológica. Árvore do conhecimento: Uva para processamento. Disponível em:

$<$ https://www.agencia.cnptia.embrapa.br/gest or/uva_para_processamento/arvore/CONT000 g5f8cou802wx5ok0bb4szwyx060i6.html>. Acesso em: 20 dez. 2015.

CAMARGO, U.A. (Rio Grande do Sul). Embrapa. Cadastro Vitícola: III Cultivares. 2005 - 2007. Disponível em:

<http://www.cnpuv.embrapa.br/pesquisa/cada stro/cds/2005-

2007/html/cult_ancellotta.html>. Acesso em: 20 dez. 2015.

DUTCOSKY, S. D. Análise Sensorial de Alimentos. 3. ed. Curitiba: Champagnat, 2011. 426p.

FROTA, K.M.G.; MORGANO, M.A.; SILVA, M.G.; ARAÚJO, M.A.M.; MOREIRAARAÚJO, R.S.R. Utilização da farinha de feijão-caupi (Vigna unguiculata $\mathrm{L}$. Walp) na elaboração de produtos de panificação. Ciência e Tecnologia de Alimentos, Campinas, v. 30, p. 16-22, 2009.

FERREIRA, J.C.; MATA, M.E.R.M.C.; BRAGA, M.E.D. Análise sensorial da polpa de umbu submetida a congelamento inicial em temperaturas criogênicas e armazenadas em câmaras frigoríficas. Revista Brasileira de Produtos Agroindustriais, Campina Grande, v. 2, n. 1, p. 7-17, 2000.

FERREIRA, L.F.D. Obtenção e caracterização de farinha de bagaço de uva e sua utilização em cereais matinais expandidos. 157p. Tese (Doutorado) Universidade Federal de Viçosa, Viçosa, 2010.
FREITAS, D.G.C.; MORETTI, R.H. Caracterização e avaliação sensorial de barra de cereais funcional de alto teor protéico e vitamínico. Ciência e Tecnologia de Alimentos., v.2, n.26, p.318-324, 2006.

GRUZ, A.P.G.; SOUSA, C.G.S.; TORRES, A.G.; FREITAS, S.P.; CABRAL, L.M.C. Recuperação de compostos bioativos a partir do bagaço de uva. Revista Brasileira

Fruticultura, Jaboticabal, v. 35, n. 4, p. 1147-1157, 2013.

LAFKA, T.I.; SINANOGLOU, V.; LAZOS, E.S. On the extraction and antioxidant activity of phenolic compounds from winery wastes. Food Chemistry, v.104, n. 3, p.1206-1214, 2007.

LOULI, V.; RAGOUSSIS, N.; MAGOULAS, $\mathrm{K}$. Recovery of phenolic antioxidants from wine industry by-products. Bioresource Technology, v.2, n. 92, p.201-208, 2004.

MAKRIS, D.P.; BOSKOU, G.; ANDRIKOPOULOS, N.K. Recovery of antioxidant phenolics from white vinification solid by-products employing water/ethanol mixtures. Bioresource Technology, v.98, n. 15, p.2963-2967, 2007.

NASCIMENTO, M.R.F.; WANG, S.H.; ASCHERI, J.L.R. Características sensoriais de donuts preparados com farinha de trigo e soja (80:20) extrusadas em diferentes parâmetros de extrusão. Alimentos e Nutrição, Araraquara, v. 20, n. 2, p. 247-256, 2009.

NATIVIDADE, M.M.P.; LIMA, L.C.O.; PINHEIRO, A.C.M. Desenvolvimento, caracterização e aplicação tecnológica de farinhas elaboradas com resíduos da produção de suco de uva. 2010. $202 \mathrm{f}$. Dissertação (Mestrado) - Curso de Ciências dos Alimentos, Universidade Federal de Lavras, Lavras, 2010. Cap. 3.

NÚCLEO DE ESTUDO E PESQUISAS EM ALIMENTAÇÃO - UNICAMP. Tabela 
Brasileira de Composição de Alimentos. 4. ed. Campinas: Fodepal, 2011. 161 p.

PAVANELLI, A.O.; CICHELLO, M.S.; PALMA, E.J. Emulsificantes como agentes deaeração em bolos. Oxiteno S/A Indústria e Comércio, ART AL004 06/00, 2000.

PINELO, M.; FABBRO, P.D.; MANZOCCO, L.; NUNEZ, M.J.; NICOLI, M.C.

Optimization of continuous phenol extraction from Vitis vinifera by products. Food

Chemistry, v.92, n. 1, p.109-117, 2005.

PIOVESANA, A.; BUENO, M.M.; KLAJN, V.M. Elaboração e aceitabilidade de biscoitos enriquecidos com aveia e farinha de bagaço de uva. Food Technology, v.16, n.1, p.68-72.

OLIVEIRA, L.T.; VELOSO, J.C.R.; TERANORTIZ, G.P. Caracterização físicoquimica da farinha de semente e casca de uva. II Semana de Ciência e Tecnologia do IFMG campus Bambuí e II Jornada Científica. 2009.

ROCHA, M.S.; FIGUEIREDO, R.W.; ARAÚJO, M.A.M.; ARAÚJO, R.S.R.M. Caracterização físico-química e atividade antioxidante (in vtitro) de frutos do cerrado piauiense. Revista Brasileira de

Fruticultura, Jaboticabal, v.35, n.4, p.933941, dez. 2013.

ROCKENBACH, I.I. Compostos Fenólicos, Ácidos Graxos e Capacidade Antioxidante do Bagaço da Vinificação de Uvas Tintas (Vitis vinifera e Vitis labrusca). 2008. $112 \mathrm{f}$. Dissertação (Mestrado em Ciência dos Alimentos) - Universidade Federal de Santa Catarina, Florianópolis, 2008.

SANCHES, M.; SALAY, E. Alimentação fora do domicílio de consumidores do município de Campinas, São Paulo. Revista de Nutrição, Campinas, v. 24, n. 2, p.295304, mar. 2011.

SOARES M.; WELTER L.; KUSKOSKI

E.M.; GOZAGA L.; FETT R. Compostos fenólicos e atividade antioxidante da casca de uvas Niágara e Isabel. Revista Brasileira de Fruticultura, Jaboticabal, v. 30, n.1, p.59-64, mar. 2008.

SPANGHERO, M.; SALEM, A.Z.M.;

ROBINSOND, P.H. Chemical composition, including secondary metabolites, and rumen fermentability of seeds and pulp of Californian (USA) and Italian grape pomaces. Animal Feed Science and Technology, v.152, n. 3-4, p.243-255, 2009.

\section{TEIXEIRA A. H. C. Cultivo da}

Videira: Aspectos agrometeorológicos da cultura da videira. 2010. Disponível em:

$<$ http://sistemasdeproducao.cnptia.embrapa.br /FontesHTML/Uva/

CultivodaVideira_2ed/clima.html $>$. Acesso em: 16 nov. 2014.

TEIXEIRA, E.; MEINERT, E.; BARBETTA, P.A. Análise sensorial dos alimentos.

Florianópolis: UFSC, 1987. 180 p.

TEIXEIRA, L.V. Análise Sensorial na Indústria de Alimentos. Revista do Instituto de Laticínios Cândido Tostes, [s. I.], v. 366, n. 64, p.12-21, jan. 2009. 